magnets are another business) usually of fer custom-built designs intended to suit the needs of people in academic or industrial laboratories in need of some predetermined configuration of magnetic field. As with NMR, from any one of these applications might spring some new range of off-the-shelf superconducting magnets.

Oxford Instruments, one of the principal suppliers, says that it will consider ad hoc proposals from potential academic customers, but will not in the first instance produce a detailed estimate of the cost of building a new design. Rather, it will undertake to produce information sufficient to sustain a research grant application, and will produce a final estimate of cost in return for a fee refundable against a firm order when the application has been successful.

Whether this esoteric business will - or would be - transformed by the development of superconducting materials with transition temperatures substantially above the boiling point of liquid helium is another matter. The chief benefit, as things are, would be quantitative: helium consumption would be reduced. But if somebody were to construct a material superconducting at liquid nitrogen temperatures, a whole new ballpark would be accessible...

\section{High-energy physics rides on flux}

THERE is a real sense in which the development of high-energy physics in the past half century has been sustained by the technology of the magnet builders. Although the first important landmark in the artificial manipulation of nuclear structure - the use of $300 \mathrm{MeV}$ protons for the disintegration of nuclei by Cockcroft and Walton in 1931 - was made possible by means of an electrostatic accelerator, E.O. Lawrence was even then building the first cyclotron at Berkeley. By the time, in 1935 or so, that the limits of electrostatic acceleration had been reached, working cyclotrons were all the rage.

The principle of these machines is simple enough. The path of a charged particle travelling in a magnetic field will be curved, but no kinetic energy will be lost. If the magnetic field is sufficiently extensive, the particle will travel in a closed path, thus increasing the length of time for which other means of accelerating it can do their work.

The cyclotrons which in the 1930s followed the Berkeley design were the simplest of all accelerating machines. The principle is simply to construct a substantial volume of uniform magnetic field between the poles of an electromagnet, to provide a source of charged particles at the centre of the arrangement and to arrange for some means of adding to the kinetic energy of the charged particles as they traverse closed orbits between the poles of the source magnet.

Cyclotrons have functioned since the

\title{
Cold comfort at room temperature
}

SUPERCONDUCTIVITY is the next best thing to perpetual motion: an electrical current once established in a superconducting circuit will keep going indefinitely. So is it not mere misfortune that the only circumstances in which known materials can be made superconducting are those involving temperatures in the liquid helium range, below $4.1 \mathrm{~K}$ or thereabouts?

After the best part of a decade, the hopes first fostered by the empirical discovery that suitably constructed linear polymer materials have electronic conduction bands and are indeed capable of conducting electricity might make possible the design of molecules exhibiting superconductivity at room temperatures. In reality, painful experience has shown that superconductivity is doggedly a low-temperature phenomenon, involving lattice or molecular vibrations (phonons) and electron movement in a cooperative transition from a disordered to an ordered state. Room-temperature superconductivity is likely always to lie in the future.

For practical purposes, it is a sufficient but still daunting goal to find materials superconducting at the temperature of liquid nitrogen, the common outer refrigerant in superconducting cooling systems.

The record so far is a transition temperature of $21 \mathrm{~K}$ for a version of $\mathrm{Nb}_{3} \mathrm{Ge}$ in which germanium atoms are partly replaced by atoms of aluminium. (This is a true superconductor of Type II, able to sustain high magnetic fields without losing its essential properties, but metallurgically hard to work with.) As things are, such interest in the search for improved superconductors centres on the sulphides of molybdenum doped with rare-earth elements as substitutes for $\mathrm{Mb}$.

In practice, even unexpectedly rapid progress in the search for superconductors with higher transition temperatures would only slowly transform the technology of making superconducting magnets. In practice the improved superconductors now coming into use are metallurgically almost impossible. They function as planned only if pure and stoichiometrically exact. It will be some time before it is a matter of routine to wind kilometres of wire on some form and call the result a magnet.

the Second World War) is a formidable undertaking.

For one thing, the sheer bulk of the early 1930s because the frequency with which a charged particle traverses its closed orbit in a magnetic field is independent of the dimensions of the orbit and simply a function of the characteristics of the particle and the strength of the field $(B)$. Thus the frequency of rotation is given by the familiar relationship $e B / 2 \pi m$, where $e$ and $m$ are the charge and mass of the particles.

Since the beginning, cyclotrons have been no better and no worse than the design of their magnets. Since the first machine at Berkeley, the standard procedure has been to accelerate charged particles by means of alternating electrostatic fields within a pair of semicircular hollow conducting surfaces shaped so that, when put together, they would constitute a hollow pancake-shaped structure - and which, when separate, are know as "dees". The two halves of this structure are fed with radiofrequency power at the characteristic frequency of the cyclotron (a function only of $B$ and the particle concerned).

One obvious difficulty with which the magnet designers have had since the beginning to contend is the sheer physical problem of shaping the magnetic field appropriately. Since charged particles spiral outwards from the central point at which they are injected, the field must be for practical purposes uniform over the maximal orbits followed by the particles. The construction of electromagnets spanning close on $2 \mathrm{~m}$ (such as with the 184-inch cyclotron built at Berkeley after magnetic circuit required to provide a return path for the magnetic field between the poles of the electromagnet where the action is is almost a feat of mechanical engineering in its own right. For another, the shaping of the pole pieces (usually machined from cast or forged blocks of soft iron) is a complicated process of balancing the loss of flux through the sides of tapering circular endpieces against the need for uniformity of field.

Throughout the 1930s, the construction of cyclotron magnets was almost a black art. Once a machine had been built, the designers of the magnet would be required to fiddle around with the placing of shims near the poles of the magnets to improve the characteristics of the field, its uniformity in particular. Even the first cyclotrons, however, were blessed with one important built-in benefit - because, between the poles of an electromagnet, flux lines unavoidably become more distant from each other, displacements of circulating charged particles towards one pole or another are discouraged by the mirroring effect thereby produced.

For practical purposes, cyclotrons have been used for accelerating protons and heavier ions, with maximum energy (determined by the area of the space between the pole pieces) measured in tens of $\mathrm{MeV}$. The acceleration of electrons to comparable and greater energies is best accomplished by means of linear accelerators (most spectacularly by the two-mile accelerator at Stanford, 